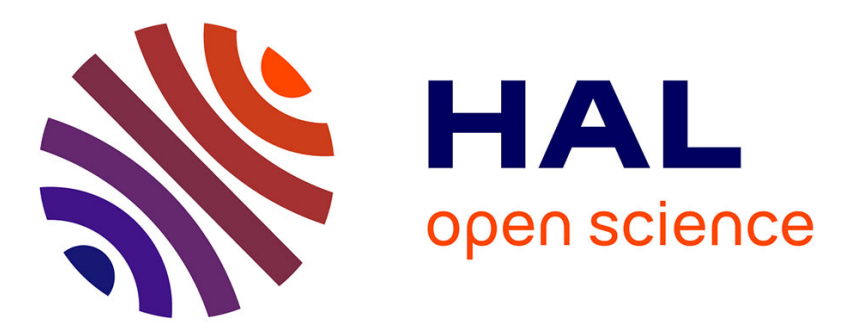

\title{
Using polarization diversity in the detection of small discontinuities by an ultra-wide band ground-penetrating radar
}

Florence Sagnard, Elias Tebchrany

\section{- To cite this version:}

Florence Sagnard, Elias Tebchrany. Using polarization diversity in the detection of small discontinuities by an ultra-wide band ground-penetrating radar. Measurement - Journal of the International Measurement Confederation (IMEKO), 2015, 61, 26p. 10.1016/j.measurement.2014.10.035 . hal01658534

\section{HAL Id: hal-01658534 \\ https://hal.science/hal-01658534}

Submitted on 7 Dec 2017

HAL is a multi-disciplinary open access archive for the deposit and dissemination of scientific research documents, whether they are published or not. The documents may come from teaching and research institutions in France or abroad, or from public or private research centers.
L'archive ouverte pluridisciplinaire $\mathbf{H A L}$, est destinée au dépôt et à la diffusion de documents scientifiques de niveau recherche, publiés ou non, émanant des établissements d'enseignement et de recherche français ou étrangers, des laboratoires publics ou privés. 


\section{Accepted Manuscript}

Using polarization diversity in the detection of small discontinuities by an ultrawide band ground-penetrating radar

Measurement

Florence Sagnard, Elias Tebchrany

PII:

S0263-2241(14)00504-1

DOI: http://dx.doi.org/10.1016/j.measurement.2014.10.035

Reference: MEASUR 3094

To appear in:

Measurement

Received Date: $\quad 1$ July 2013

Revised Date: $\quad 20$ October 2014

Accepted Date: $\quad 23$ October 2014

Please cite this article as: F. Sagnard, E. Tebchrany, Using polarization diversity in the detection of small discontinuities by an ultra-wide band ground-penetrating radar, Measurement (2014), doi: http://dx.doi.org/10.1016/ j.measurement.2014.10.035

This is a PDF file of an unedited manuscript that has been accepted for publication. As a service to our customers we are providing this early version of the manuscript. The manuscript will undergo copyediting, typesetting, and review of the resulting proof before it is published in its final form. Please note that during the production process errors may be discovered which could affect the content, and all legal disclaimers that apply to the journal pertain. 


\title{
Using polarization diversity in the detection of small discontinuities by an ultra-wide band ground-penetrating radar
}

Florence Sagnard, and Elias Tebchrany University Paris-Est, IFSTTAR, Department MACS, 14-20, Bd Newton, 77420 Champs-sur-Marne, France Phone : +33181668491

florence.sagnard@ifsttar.fr, elias.tebchrany@ifsttar.fr

\begin{abstract}
A ground-penetrating radar (GPR) has been developed for civil engineering applications. Ultra-wide band (UWB) bowtie slot antennas operating at frequencies from $460 \mathrm{MHz}$ to beyond $4 \mathrm{GHz}$ have been designed to be integrated in a ground-penetrating radar (GPR) positioned very close to the soil surface. FDTD modeling has allowed to define the optimal geometrical parameters associated with the ground-coupled radar system that has been studied in two polarization configurations TE and TM. The ability of the GPR system in detecting small discontinuities using polarization diversity has been analyzed considering several buried canonical objects with different dielectric characteristics. Measurements in a sand box have been compared to full-wave simulations.
\end{abstract}

\section{Introduction}

Ground-penetrating radar (GPR) is a well-known non-destructive technique based on electromagnetic waves to detect and image dielectric discontinuities in the subsurface [1]. GPR applications are numerous involving archaeology, geology, civil engineering, environmental geophysics, utilities or target detection. The dielectric properties of a soil correlate with many of the mechanical, geologic and structural parameters of the constituents. The choice of the central frequency and the bandwidth of the GPR is an important issue, and depends on the particular application, mostly of the kind of object one is looking for: size of the object, its electrical properties, depth of the object, properties of the soil, etc... An ultrawide band (UWB) radar offers an opportunity to probe a soil structure using the benefit from 
both low and high frequencies and to obtain a compromise in terms of depth resolution and penetration in a single measurement.

The basic GPR system is made of a pair of parallel antennas that radiate a polarized electric field, and the co-polarization configuration is generally chosen. Thus, the GPR system probes the subsurface at a single polarization. An optimal matching of the GPR system with the soil in order to eradicate the significant reflection from the air-soil interface is achieved by positioning both antennas in close proximity to the soil interface. The application of interest is the detection of buried objects or discontinuities in civil engineering structures particularly pipes [2, 3], and defaults (cracks, joints, buried repair patches, delaminations, density changes, etc...) which can be caused by mechanical, chemical and biological causes. Because the GPR reflected signal traces issued from embedded objects result from multiple scattering both inside each object and between a given object and its environment, we have considered a simplified soil structure to interpret the radar response by comparing numerical FDTD modeling and field-experiments in the presence of well-separated canonical objects. Thus, we have designed a compact UWB GPR system using full-wave FDTD simulations to model and control its radiation characteristics. The experimental GPR system is supposed to operate in the frequency domain using a step-frequency continuous wave (SFCW) to benefit from a wide dynamic range, a low figure noise and the possibility of shaping the power spectral density. The aim of this study is to check the sensitivity of the UWB GPR for the detection of small discontinuities in the two main polarization configurations TM (Transverse Magnetic) and TE (Transverse Electric), and to extract information of dielectric characteristics from reflection hyperbolas. The comparison of theoretical and experimental B-scans has allowed to interpret the polarization phenomena involved by different discontinuities characterized by their shape and their dielectric properties.

\section{UWB GPR system}

The GPR system consists of a pair of transmitting and receiving shielded bowtie slot antennas. The antennas have been designed in our laboratory using full-wave FDTD simulations under the commercial software EMPIRE. The classical geometry of the bowtie slot antenna, as visualized in Figure 1a, has been modified to operate in an UWB from frequency $460 \mathrm{MHz}$ to beyond $4 \mathrm{GHz}$ (reflection coefficient $S_{11 d B}(f)$ less than $-10 \mathrm{~dB}$ ) and at lower frequencies as usual with a reduced size close to the A4 sheet size [4-9]. The antenna 
has been designed on a single-sided FR4 substrate $\left(h=1.5 \mathrm{~mm}, \varepsilon_{r}{ }^{\prime}=4.4 ; \tan \delta=0.01,35 \mu \mathrm{m}\right.$ copper-clad) and its overall dimensions are $W_{s}=190.4 \mathrm{~mm}$ and $L_{s}=332.2 \mathrm{~mm}$. As visualized in Figure 1b, the antenna is fed at its port by a tapered coplanar waveguide CPW line with length $W_{f}=67.02 \mathrm{~mm}$ and dimensions $a=0.4 \mathrm{~mm}$ and $b=2.8 \mathrm{~mm}$. The antenna port is supposed to be connected to a Vector Network Analyzer (VNA) via a SMA (subminiature version A) connector. To eliminate backward radiations in air induced by the close environment, and to reduce the direct coupling between transmitting and receiving antennas in the GPR system, each antenna has been partially enclosed in a perfectly conductive box opened towards the ground surface as shown in Figure 2; the conductive box (height $h_{c}=67.5$ $\mathrm{mm}$, width $\mathrm{W}_{\mathrm{c}}=231 \mathrm{~mm}$, and length $\mathrm{L}_{\mathrm{c}}=362 \mathrm{~mm}$ ) has been filled with a layered absorbing material which is supposed to progressively absorb the electromagnetic waves and to reduce multiple reflections inside the cavity. In the numerical modeling, a three-layered radar absorbing material (RAM) with a conductivity profile characterized by the power law $\sigma=10^{n-3} S . m^{-1}$ ( $\mathrm{n}$ is the layer number, and $\mathrm{n}=1$ is the layer just in contact with the antenna) has been considered; such a structure is supposed to represent the five-layered RAM (150 mm-thick absorbing foam HPS 125 distributed by EUROMC) used in the experiments [10]. Thus, according to a parametric study non presented here, the characteristics of the absorbing layers are finally: $\varepsilon_{1,2,3}=1.5, \quad \sigma_{1}=0.01 \mathrm{~S} . \mathrm{m}^{-1} ; e_{1}=24.2 \mathrm{~mm} ; \sigma_{2}=0.1 \mathrm{~S} . \mathrm{m}^{-1} ; e_{2}=29.3 \mathrm{~mm}$ and $\sigma_{3}=1 S \cdot \mathrm{m}^{-1} ; e_{3}=14 \mathrm{~mm}$. The printed face of the antenna, positioned very close to the soil at an elevation $h_{s}$ around $10 \mathrm{~mm}$ to consider potential irregularities on the soil surface, has been protected by a thin PVC sheet.

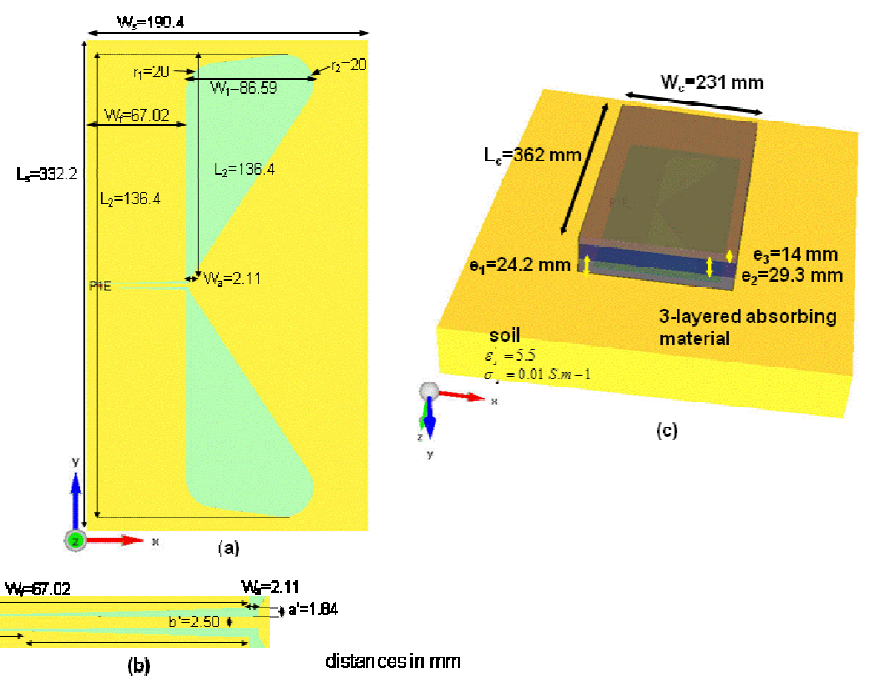

Figure 1 
Measurements have been made in the frequency domain, in the band $[0.05 ; 4] \mathrm{GHz}(1601$ samples, Intermediate Frequency (IF) bandwidth $500 \mathrm{~Hz}$ ) using a portable VNA ANRITSU MS 2026B. A full two ports calibration has been made with $2 \mathrm{~m}$ length radiofrequency coaxial cables. The measured reflection coefficients $S_{11}(f)$ associated with a single antenna positioned on a wet sandy soil have been compared to FDTD simulated results considering several real permittivity values of the soil. We observe from Figure $2 \mathrm{a}$ a slight difference between simulations and measurements at the lower frequencies corresponding to $-10 \mathrm{~dB}$. Moreover, we remark that theoretical results associated with the shielded antenna on a soil with relative real permittivity value $\varepsilon_{s}^{\prime}=3.5$ (conductivity $\sigma_{s}=0.01 S . \mathrm{m}^{-1}$ ) appear the closest to the measurements. This permittivity estimation will be confirmed later by offset measurements using the GPR system. The E-field distributions in the TE and TM planes $(\mathrm{f}=800 \mathrm{MHz})$ visualized in Figure $2 \mathrm{~b}$ in proximity to the unshielded antenna positioned on a soil interface $\left(\varepsilon_{s}^{\prime}=3.5, \sigma_{s}=0.01 S . m^{-1}, h_{s}=10 \mathrm{~mm}\right)$ highlights that the TM plane has null radiating energy at particular angles [11]; this phenomenon is also observed when the antenna is shielded as shown in Figure $2 \mathrm{c}$ at the frequency $800 \mathrm{MHz}$.

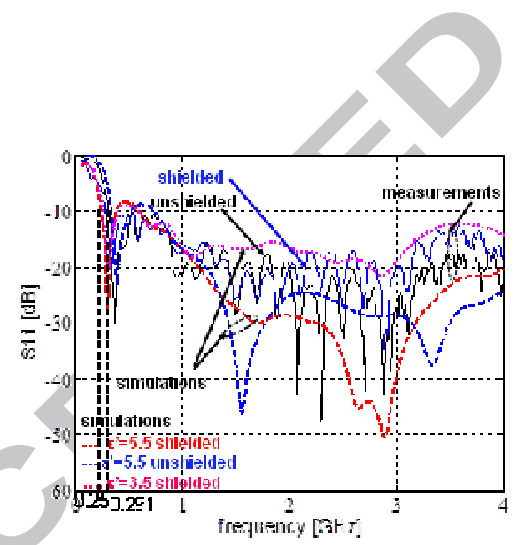

(a)

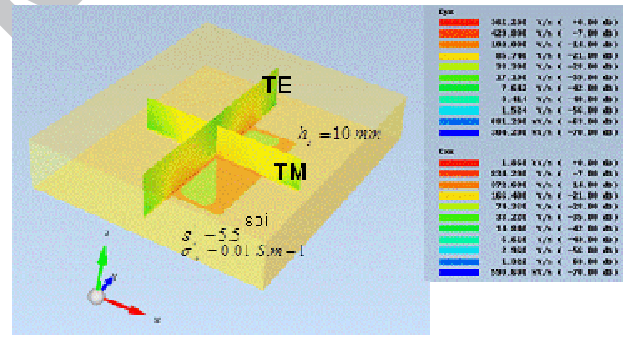

(b)

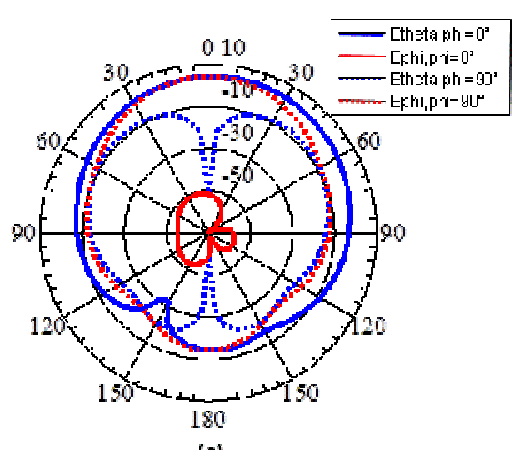

(c)

Figure 2

To acquire GPR linear profiles (B-scans), two symmetric antenna configurations have allowed to consider two polarizations: the parallel configuration (or end-fire, transverse 
magnetic mode TE, the opposite situation of a dipole antenna) with both antennas aligned along their larger dimension (see Figure 3a), and the mirror configuration (or broadside, transverse electric mode TM) with both antennas facing each other symmetrically along their larger dimension (see Figure 3b). For each configuration, the offset value between antennas results from a compromise between a weak direct path amplitude (low transmitting coefficient $\left.S_{21}(f)\right)$ and a sufficient high signal amplitude issued from reflections inside the soil induced by a buried object or a dielectric contrast. Offset measurements (Wide Angle Reflection and Refraction, WARR profiles) have been made in the range $[50 ; 1000] \mathrm{mm}$ in the parallel and mirror configurations (see Figure 4) on a wet sand. The comparison between theoretical (soil characteristics $\varepsilon_{s}^{\prime}=5.5 ; \sigma_{s}=0.01 S . m^{-1}$ see ) and experimental results in Figures $5 \mathrm{a}, 5 \mathrm{c}$ and Figure $6 \mathrm{a}, 6 \mathrm{c}$ highlights a higher coupling between both antennas in the measurements with a $S_{21}(f)$ amplitude difference around $12 \mathrm{~dB}$ for frequencies lower than $1.5 \mathrm{GHz}$; however, it has to be considered that the dielectric real permittivity of the wet sand has been a priori estimated less than 5.5. It appears from the simulations that a higher coupling at a given offset particularly below $1 \mathrm{GHz}$ is observed in the mirror configuration, that can be explained by the lower lateral dimension of each antenna in this configuration; this coupling phenomenon does not appear so marked in the measurements. Thus, according to theoretical and experimental results, the antenna offset will be then defined to $60 \mathrm{~mm}$ that allows to obtain a compact system with a reduced coupling at lower frequencies.

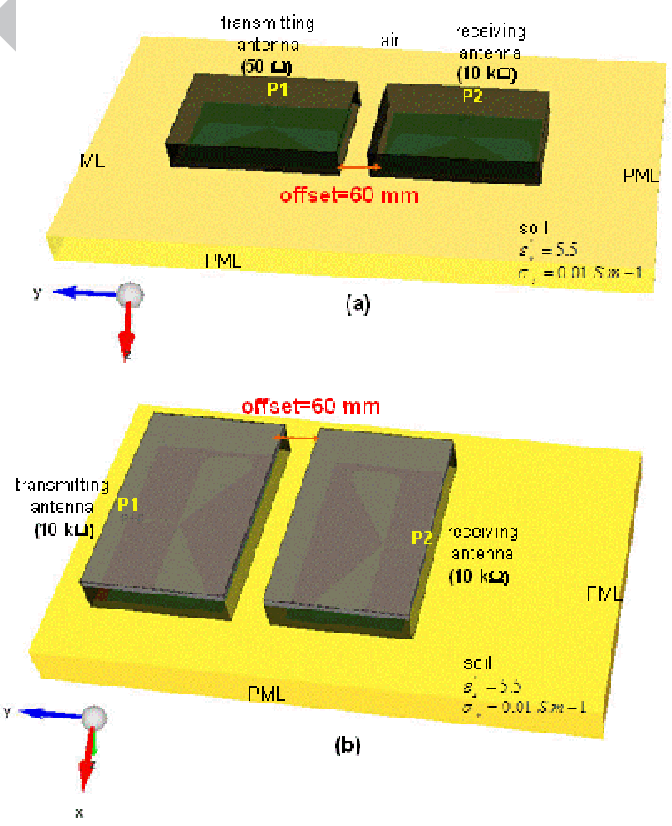

Figure 3 


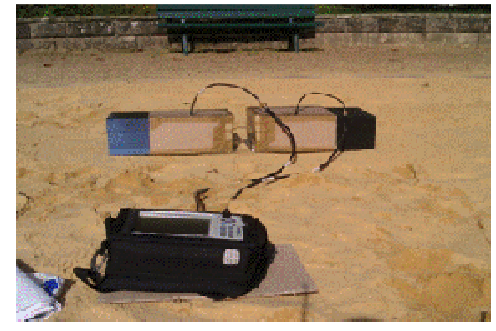

(a)

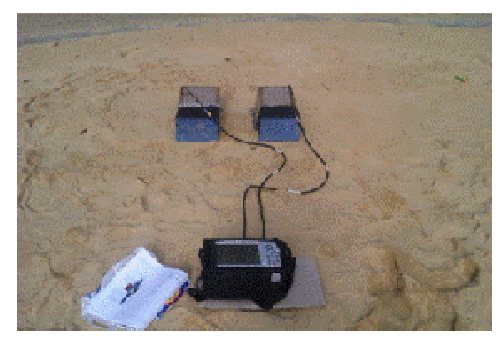

(b)

Figure 4

From the complex transmission coefficients $\tilde{S}_{21}$ measured in the frequency band [0.05; 4] GHz using a VNA, we have obtained the WARR profiles in the time domain. Thus, an apodisation of each frequency curve associated with an offset has been made to smoothly extend the band from 4 to $9 \mathrm{GHz}$. The excitation signal used, that has also been defined in FDTD simulations, is the first derivative of the Gaussian function, with duration $0.5 \mathrm{~ns}$ corresponding to $99 \%$ of the total energy $(\mathrm{t} 0=0.33 \mathrm{~ns})$. The product of the spectrum of this excitation signal with the measured $\tilde{S}_{21}$ has been performed to further calculate the inverse Fourier transform (IFFT) and obtain time data. The calibration of the time zero in the measurements has been made using the theoretical air wave time arrival. We remark that the experimental time profiles (distance step of $50 \mathrm{~mm}$ ), visualized in Figures 6b, 6d, compare satisfactorily to the theoretical profiles in Figures $5 \mathrm{~b}, 5 \mathrm{~d}$. The travel time curves $t(y)$ associated with the direct wave in air (linear) and the transmitted wave (hyperbolic) in the soil have been plotted in the profiles $\left(\varepsilon_{s}^{\prime}=3.5\right.$ in the measurements and $\varepsilon_{s}^{\prime}=5.5$ in the simulations). Depending of the velocity of the medium $v=c / \sqrt{\varepsilon_{s}^{\prime}} \quad\left(\varepsilon_{s}^{\prime \prime} \ll<\varepsilon_{s}^{\prime}\right)$, the arrival time $t(y)$ is expressed as a function of the horizontal position $y$ of the GPR system as follows:

$$
t^{2}(y)=t^{2}(0)+y^{2} / v^{2}
$$

In the measurements, the bottom of the sand box associated with an arrival time close to $6 \mathrm{~ns}$ corresponds to a depth around $470 \mathrm{~mm}$. 

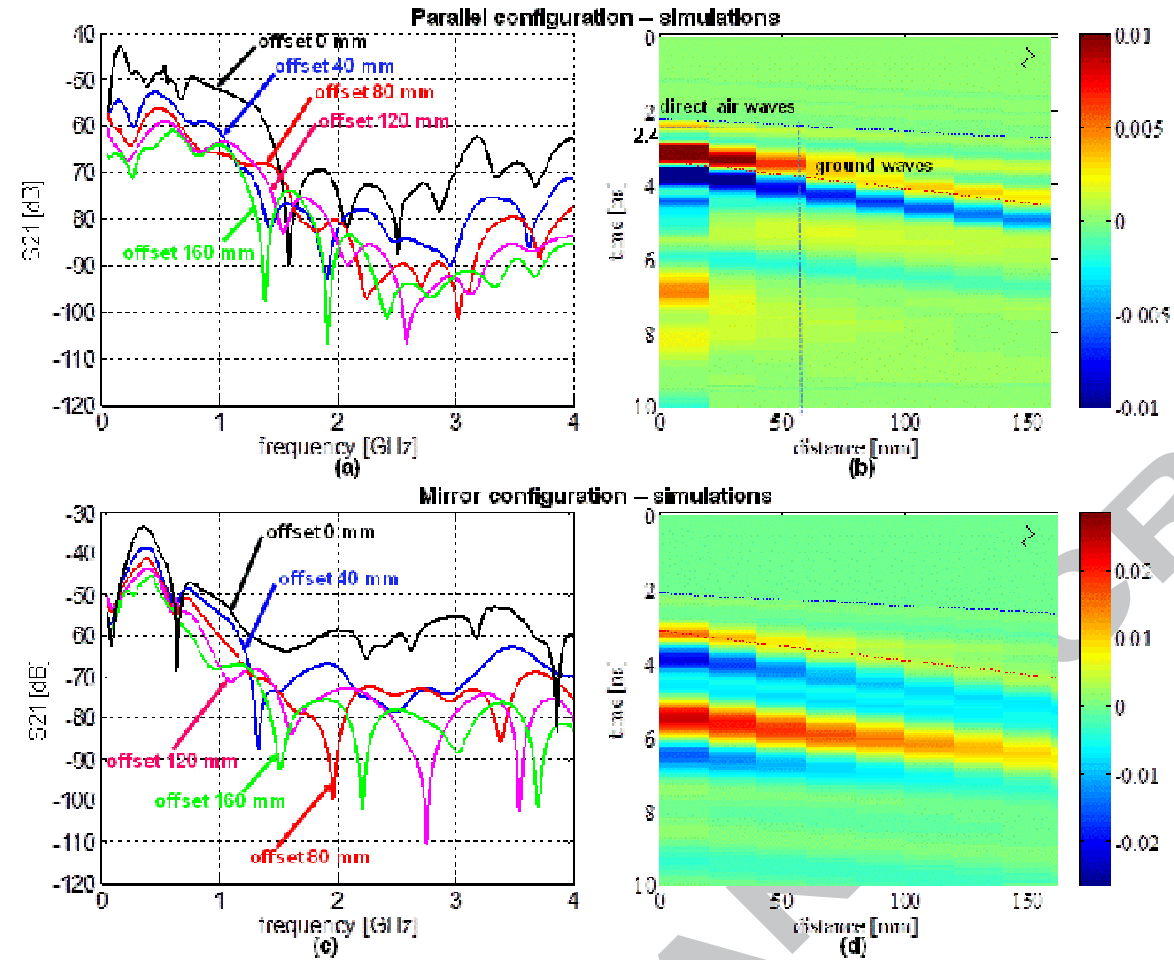

Figure 5

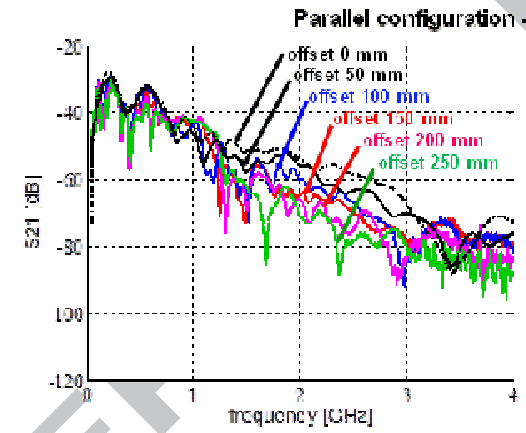

(a)

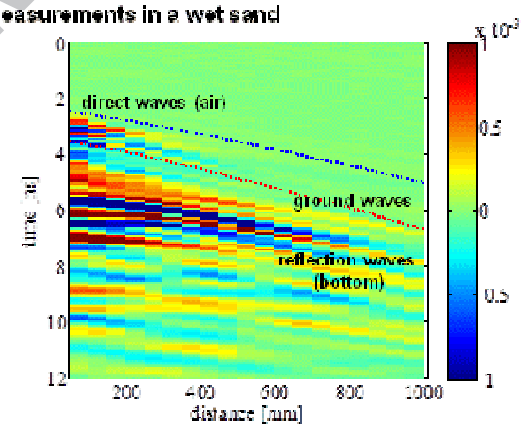

(b)

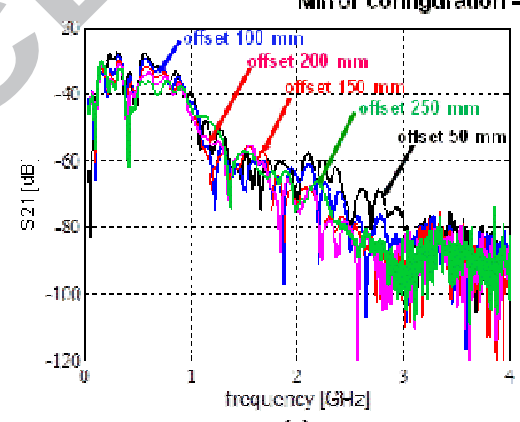

(c)

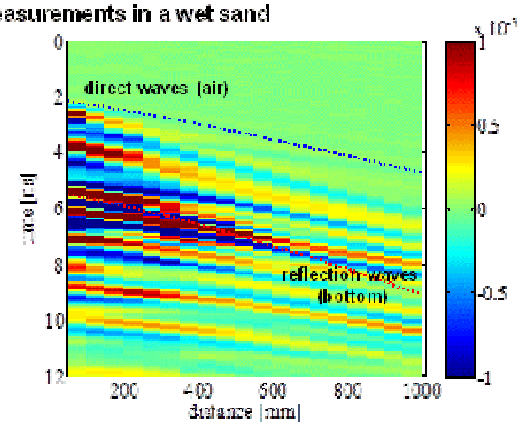

(d)

Figure 6 


\section{Theoretical aspects in discontinuity detection}

The present study is particularly focused on the detection of small canonical discontinuities (lateral dimensions less than $25 \mathrm{~mm}$ ) to further consider defaults in civil engineering structures. Such discontinuities allow to evaluate the GPR system in both polarization configurations. The antenna offset has been defined to $60 \mathrm{~mm}$ (common offset mode (COM) configuration), thus the distances between transmitting and receiving ports is $S R=422 \mathrm{~mm}$ in the parallel configuration, and $S R=291 \mathrm{~mm}$ in the mirror configuration.

\subsection{Analytical modeling}

When the GPR system moves along a straight line on the soil surface a discrete discontinuity induces reflected waves when it is under the antenna footprint. Because the antenna footprint has the shape of a wide cone, the target is imaged not only when the GPR system is immediately above, but also when the GPR is approaching several centimeters from the target. This leads to a defocalised signature with a hyperbolic shape. The two-way travel time is easily modeled by analytical relations associated with the far-field zone (ray path hypothesis) in the case of a buried cylindrical pipe. Such a modeling helps to interpret the different object signatures observed in both polarizations in a radar profile that are induced by the dielectric contrast between the surrounding medium and the object. Thus, the hyperbolic signature is formulated using a geometric model based on the Pythagorean theorem, and we have considered the center-to-center antenna offset $S R$.

The buried cylindrical object assumed infinitely long and conductive is characterized by : a radius $R$, a horizontal location at abscissa $y_{0}$ and a depth $d$ under the soil surface. The displacement of the center axe of the radar along axis Oy is noted $y_{i}$, and the signal issued from the reflection on the object arrives at time $t_{i}$.

According to Figure 7a, the travel-time equations write as follows [12]:

$$
\begin{aligned}
& \left\{\begin{array}{l}
y_{T}=y_{i}-S R / 2 \\
y_{R}=y_{i}+S R / 2
\end{array}\right. \\
& \left\{\begin{array}{l}
T_{T x 2 \text { targ } e t}=\left[\left(y_{0}-y_{T}\right)^{2}+(d+R)^{2}\right]^{0.5}-R \\
T_{t \text { arget } 2 R x}=\left[\left(y_{0}-y_{R}\right)^{2}+(d+R)^{2}\right.
\end{array}\right]^{0.5}-R
\end{aligned}
$$

The generalized hyperbola equation $\left(y_{i}, t_{i}\right)$ including the antenna offset is expressed by:

$$
t_{i}=\left(T_{T x 2 \text { target }}+T_{\text {target2Rx }}\right) / v
$$

Thus, the hyperbola depends on five parameters $\left(S R, x_{0}, d, R, v\right)$. First analysis of measured Bscans with distinguishable hyperbola signatures can be performed using this modeling. In the 
case of reproducible background clutter and antenna coupling, there influence can be significantly reduced by removing the median value at each time sample. Afterwards, the hyperbola points $\left(y_{i, \text { meas }}, t_{i, \text { meas }}\right)$ have been extracted by detecting the maximum or the minimum along each column in a selected area. Then, the minimum of the sum of squares of the distances between theoretical and measured data can be solved using the Gauss-Newton method as follows:

$$
F=\sum_{i=1}^{n}\left(y_{i, \text { theo }}-y_{i, \text { meas }}\right)^{2}
$$

Some constraints relative to the parameter ranges have been added in the algorithm to better drive the solution.
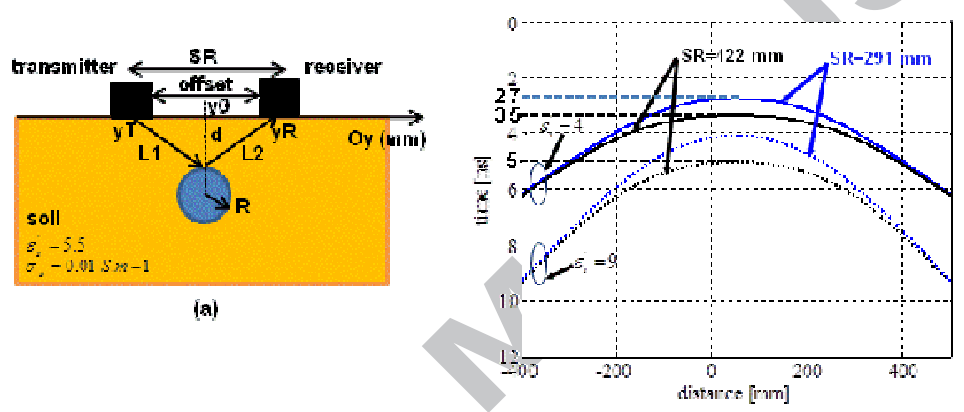

(b)

Figure 7

Considering a cylindrical pipe with radius $R=10 \mathrm{~mm}$ buried at a depth $d=150 \mathrm{~mm}$ and two soil real permitivities $\varepsilon_{s}^{\prime}=4$ and $9\left(\sigma_{s}=0\right)$, the hyperbola travel times associated with both antenna configurations, parallel and mirror, have been plotted in Figure 7b. For both soil velocities, hyperbola widths corresponding to $1.05 t_{\text {apex }}$ have been respectively estimated to 240 and $180 \mathrm{~mm}$; they appear close to $S R / 2$. Moreover, we remark that the time difference between both polarizations is close to $1 \mathrm{~ns}$ and $1.8 \mathrm{~ns}$ for the soil permittivities $\varepsilon_{s}^{\prime}=4$ and 9 respectively.

The influence of the dielectric characteristics of the cylinder (index d) illuminated by a electromagnetic (EM) polarized plane wave is described by the scattering theory [13, 14]. The backscattered fields $\left(\vec{E}_{s}, \vec{H}_{s}\right)$ depend strongly on the orientation of the cylinder relative to the antennas, its dielectric properties, and the radius of the cylinder compared to the wavelength $\lambda_{0}$ of the surrounding medium (index 0 ). The scattering properties of a long cylinder has been described by the Bessel and Hankel functions. The scattering width (SW) is the equivalent 
area proportional to the apparent size of the target (in the far-field zone) that scatters the field relative to the incident power in a given direction $\phi$. Four expressions associated with SW consider both TE and TM polarizations and the case of a dielectric or a conductive cylinder as follows:

- TE polarization :

Conductive cylinder $S W=\frac{2 \lambda_{0}}{\pi}\left|\sum_{n=0}^{+\infty} \varepsilon_{n} \frac{J_{n}^{\prime}\left(\beta_{0} R\right)}{H_{n}^{(2)^{\prime}}\left(\beta_{0} R\right)} \cos (n \phi)\right|^{2}$

Dielectric cylinder:

$$
S W=\frac{2 \lambda_{0}}{\pi}\left|\sum_{n=0}^{+\infty} \varepsilon_{n} \frac{\eta_{d} J_{n}^{\prime}\left(\beta_{d} R\right) J_{n}\left(\beta_{0} R\right)-\eta_{0} J_{n}^{\prime}\left(\beta_{0} R\right) J_{n}\left(\beta_{d} R\right)}{\eta_{0} J_{n}\left(\beta_{d} R\right) H_{n}^{(2)^{\prime}}\left(\beta_{0} R\right)-\eta_{d} J_{n}^{\prime}\left(\beta_{d} R\right) H_{n}^{(2)}\left(\beta_{0} R\right)} \cos (n \phi)\right|^{2}
$$

- TM polarization :

Conductive cylinder $S W=\frac{2 \lambda_{0}}{\pi}\left|\sum_{n=0}^{+\infty} \varepsilon_{n} \frac{J_{n}\left(\beta_{0} R\right)}{H_{n}^{(2)}\left(\beta_{0} R\right)} \cos (n \phi)\right|^{2}$

Dielectric cylinder $S W=\frac{2 \lambda_{0}}{\pi}\left|\sum_{n=0}^{+\infty} \varepsilon_{n} \frac{\eta_{0} J_{n}^{\prime}\left(\beta_{d} R\right) J_{n}\left(\beta_{0} R\right)-\eta_{d} J_{n}^{\prime}\left(\beta_{0} R\right) J_{n}\left(\beta_{d} R\right)}{\eta_{d} J_{n}\left(\beta_{d} R\right) H_{n}^{(2)^{\prime}}\left(\beta_{0} R\right)-\eta_{0} J_{n}^{\prime}\left(\beta_{d} R\right) H_{n}^{(2)}\left(\beta_{0} R\right)} \cos (n \phi)\right|^{2}$

Where $\beta_{0}$ and $\beta_{d}$ are the wavenumbers, associated with the surrounding medium and the dielectric cylinder; they can be complex. $J_{n}\left(\beta_{0} R\right)$ is the Bessel function of the first kind of order $\mathrm{n}, J_{n}^{\prime}\left(\beta_{0} R\right)$ is its derivative, $H_{n}^{(2)}\left(\beta_{0} R\right)$ is the Hankel function of the second kind of order $\mathrm{n}$, and $H_{n}^{(2)^{\prime}}\left(\beta_{0} R\right)$ is its derivative.

The simplified modeling of the GPR system considers that the antennas, linearly polarized, are closely spaced. This results to a backscattering angle corresponding to $\phi=180^{\circ}$. In this context, before analyzing theoretical and experimental B-scans, we have studied the scattered responses SW in a very large frequency band $[0.2 ; 2] \mathrm{GHz}$ considering several pipe radii and two dielectric contrasts between the pipe and the surrounding medium. The results collected in Figures $8 \mathrm{a}$ and $8 \mathrm{~b}$, in the case of a conductive cylinder with a radius in the range $[5 ; 40]$ mm, highlight that the TM polarization (electric field parallel to pipe axis) generally leads to a higher backscattering response whatever the value of the real permittivity value $\left(\varepsilon_{0}^{\prime}=4\right.$ or 9 ; $\left.\varepsilon_{0}^{\prime \prime}=0\right)$ of the surrounding medium. We remark that the TE component oscillates under the TM component for a radius higher than $5 \mathrm{~mm}$. In general, the scattering width increases with the pipe radius and when the radius becomes large as compared to the wavelength of the medium; at $2 \mathrm{GHz}, \lambda_{0}=75 \mathrm{~mm}$ for $\varepsilon_{0}^{\prime}=4$, and $\lambda_{0}=50 \mathrm{~mm}$ for $\varepsilon_{0}^{\prime}=9$. Consequently, the TM 
polarization is preferred to detect a conductive pipe independently of its radius and the wavelength of the surrounding medium. Figures $8 \mathrm{c}$ and $8 \mathrm{~d}$ associated with a dielectric cylinder show that the TE polarization gives a higher SW when the radius value is inferior to the wavelength and when the permittivity of the pipe (here filled with air) is less than the medium permittivity $\left(\varepsilon_{0}^{\prime}=4\right)$. When the pipe is more dielectric $\left(\varepsilon_{0}^{\prime}=9\right)$ than the medium, we remark that the TM polarization is in general higher than the TE polarization. We also observe that for a radius greater than $10 \mathrm{~mm}$, TE and TM components oscillate and they can meet each other.

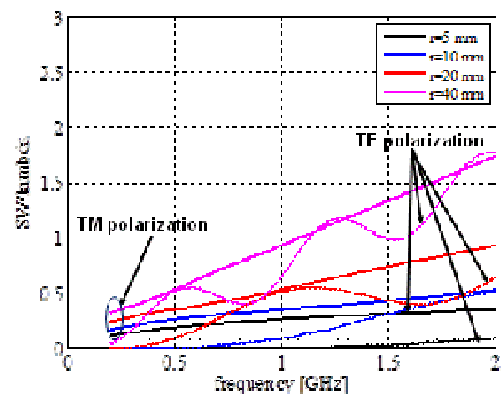

(a) conductlve cyllinder in a soll $c-1$

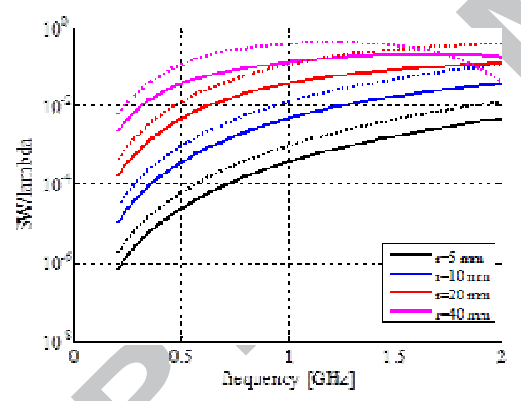

(c) dislectric cylnder $(r:-1)$ in a soll $c-1$

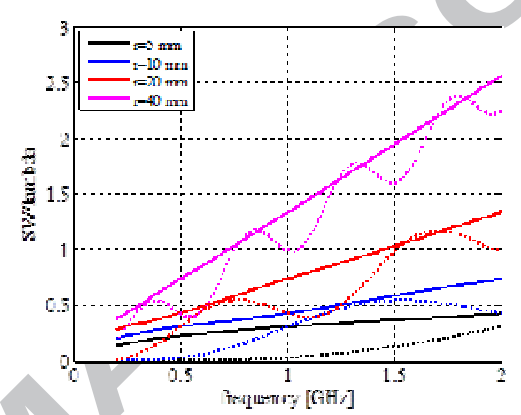

(b) conductive cylnder li a coll $s_{-}-9$

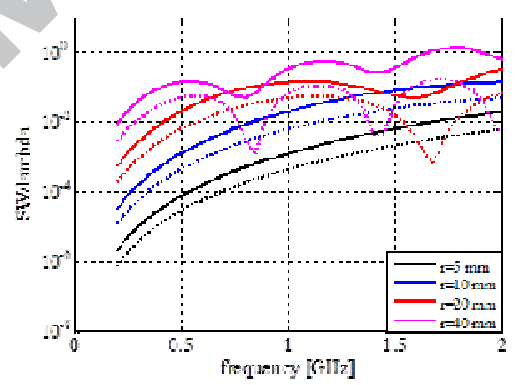

(d) delectric cylnder ( $r$ 'm) In a soll $c$ -

Figure 8

\subsection{Numerical modeling}

Full-wave simulations based on the FDTD approach considering buried canonical discontinuities have been performed to preliminary analyze radar profiles (B-scans). The moving step of the GPR system along the horizontal axis has been defined to $40 \mathrm{~mm}$, and the elevation on the ground surface is $h_{s}=10 \mathrm{~mm}$. The soil is homogeneous and includes losses expressed by its conductivity $\sigma_{s}=0.01 S . \mathrm{m}^{-1}$. The excitation current has the shape of the derivative of the Gaussian function with a time zero estimated to $0.3 \mathrm{~ns}$ and a duration (99\% of the total energy) of $0.5 \mathrm{~ns}$. 
A $5 \mathrm{~mm}$-width rectangular air-filled crack of height $75 \mathrm{~mm}$ opened towards the soil interface has been first probed in the parallel and mirror configurations, as shown in Figures 9a and 9b. The crack is centered at the position $y=-402.5 \mathrm{~mm}$, and the displacement step used in the simulations is $40 \mathrm{~mm}$. The real permittivity of the soil considered is $\varepsilon_{s}^{\prime}=5.5$. To reduce the antenna coupling expressed in the B-scans by horizontal lines having different polarities, the horizontal median value associated with each time sample has been removed as presented in Figures $9 \mathrm{c}$ and $9 \mathrm{~d}$. Thus, we remark that the TE polarization gives a more blurred image than the TM polarization that gives a sharp response. The arrival times of the direct wave has been estimated to $2.8 \mathrm{~ns}$ and $2.2 \mathrm{~ns}$ in the parallel and mirror configurations respectively, that correspond to the analytical model with the average celerity in air and in the soil. The first arrival times of the hyperbola apexes are 3.7 and $3.3 \mathrm{~ns}$ in both polarizations respectively; they correspond to the reflection at the bottom of the crack at the depth $75 \mathrm{~mm}$. There values agree with those issued from the simplified model illustrated in Figure $7 \mathrm{a}$ with an object positioned at the depth $75 \mathrm{~mm}$. Afterwards, hyperbola extraction has been performed on grayscale images. Selecting the area where the hyperbola is located, the minimum of the maximum (parallel case) or the minimum (mirror configuration) at each horizontal position has been stored. Then, the hyperbola fitting has been performed in two steps: firstly, a polynomial fitting (degree 2) using the least-square criterion has been performed on the raw data, and secondly, then a hyperbola fitting based on relation (4) has been applied on the polynomial data. This results in the fitting curves shown in Figures 9c and 9d. In general, we remark that the mirror configuration gives a higher scattering amplitude than the parallel one as shown in Figures 9c, 9d and 10a (position $y_{i}$ of the apex); this result agrees satisfactorily with the plots of Figure 8c. In this case, the probing in both polarizations can give dielectric information concerning the detected discontinuity, however it remains difficult to estimate its width. These fitting curves have been compared in Figure 10b with those associated with an open V-crack of $5 \mathrm{~mm}$ width at the soil interface with height $75 \mathrm{~mm}$. This comparison highlights that it appears difficult to distinguish in a B-scan from a rectangular and a V-crack when they are thin. 

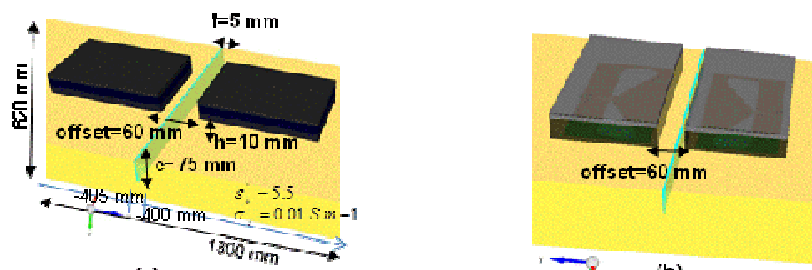

(a)
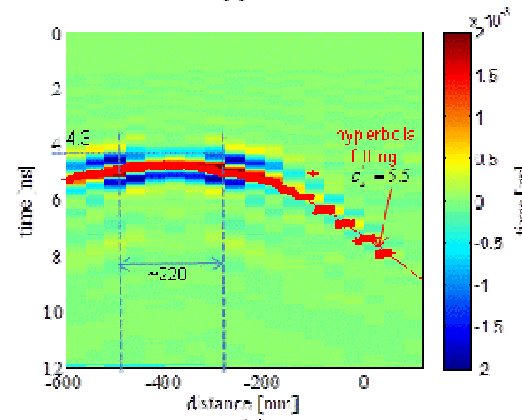

(c)

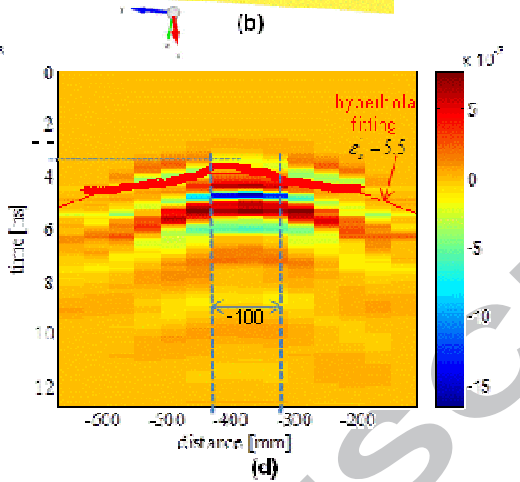

Figure 9

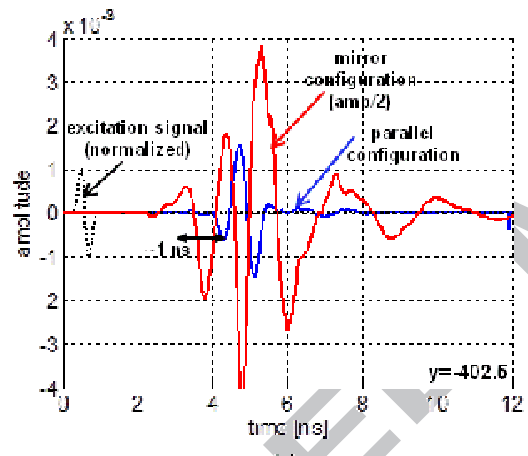

(a)

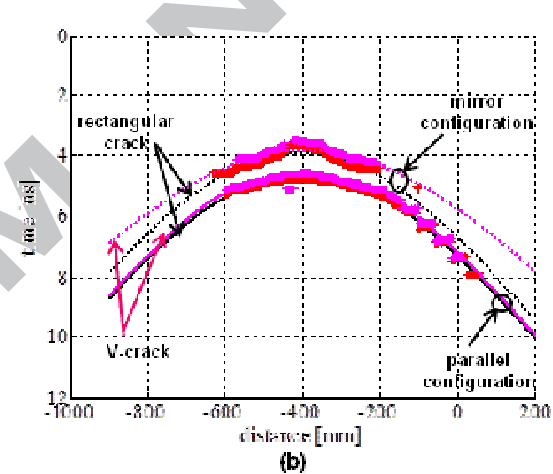

(b)

Figure 10

A conductive pipe with radius $R=10 \mathrm{~mm}$ buried at the depth $160 \mathrm{~mm}$ in a homogeneous soil

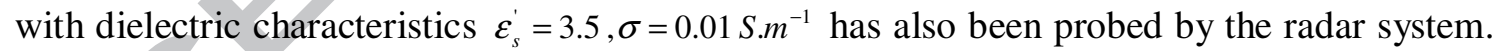
B-scans associated with the parallel and mirror configurations as visualized in Figures 11a and $11 \mathrm{~b}$ have been obtained from FDTD simulations. The arrival times from the B-scans associated with the clutter are estimated to 3 and $3.3 \mathrm{~ns}$ in the parallel and mirror configurations respectively, and the first arrival times associated with the hyperbola apexes are 4.6 and $4 \mathrm{~ns}$; the first positive peak of the incident signal arrives at $0.5 \mathrm{~ns}$. Thus, we remark a positive time difference of around $0.5 \mathrm{~ns}$ for the clutter, and $0.8 \mathrm{~ns}$ for the reflection hyperbola when comparing to the analytical model. Figures 11c and 11d highlight that the TM polarization induces a higher and sharper reflection amplitude as compared to the TE polarization. The signal at the apex in the TM polarization is characterized by a sharp and 
significant positive component and higher than the clutter, as shown in Figure 12. In the TE polarization, the positive and negative components show similar amplitudes and the signature appears more blurred. The hyperbola traces associated with the first reflection on the pipe in both polarization and calculated using the analytical relation (4) have been superimposed to the B-scans in Figures 11c and 11d; these traces follow satisfactorily the signatures observed in the calculated B-scans.

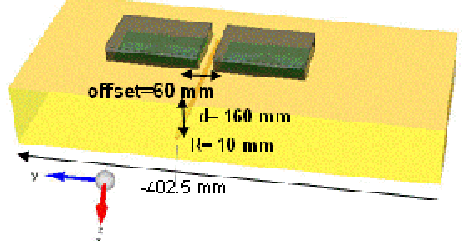

(3)

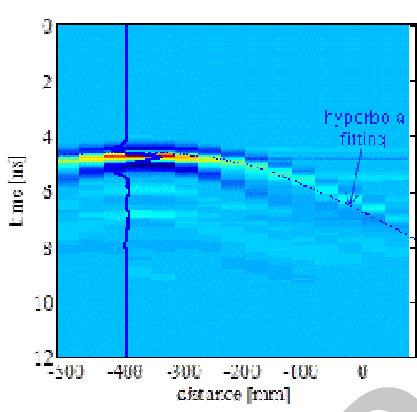

(c)

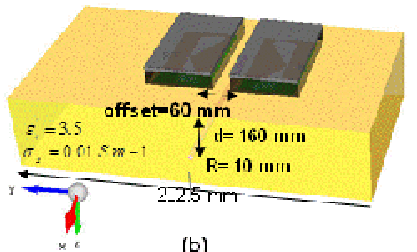

(b)

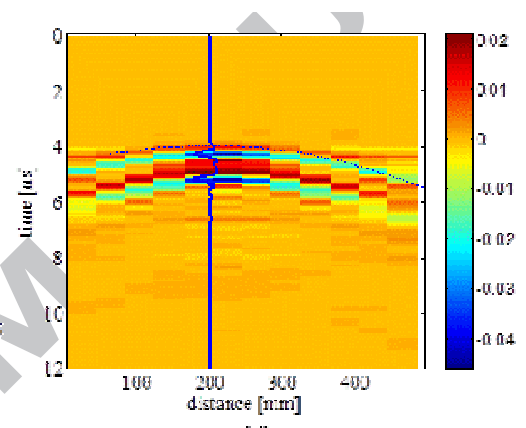

(d)

Figure 11

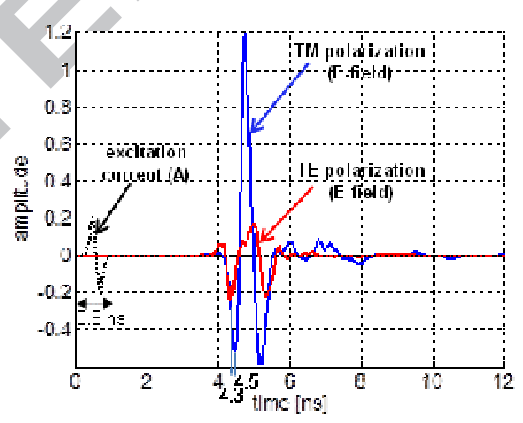

Figure 12

\section{Analysis of experimental results}

Buried canonical objects in the large sandy box of the public square Perichaux, Paris $15^{\text {th }}$ district, have been probed using the radar system developed. The sand was wet and not compacted; its dielectric permittivy has been estimated to 3.5 from the slope of the WARR profiles (see Figures $6 \mathrm{~b}$ and $6 \mathrm{~d}$ ). The time zero has been corrected according to the comparisons of WARR profiles issued from measurements and simulations (Figures 6b and 
6d). In general, a couple of canonical objects (pipe or strip) has been probed in a single Bscan to compare the signatures. Both objects have different dielectric properties, and have been buried at a depth close to $150 \mathrm{~mm}$. They have been separated by a distance around $700 \mathrm{~mm}$, and the displacement range is $[0 ; 1760] \mathrm{mm}$ with a $40 \mathrm{~mm}$ step; the position $y_{i}$ corresponds to the center of the radar system in both polarizations. The complex transmission coefficients $\tilde{S}_{21}$ have been measured in both polarizations, parallel and mirror, at an offset of $60 \mathrm{~mm}$ in the frequency domain $[0.05 ; 4] \mathrm{GHz}$ (1601 samples) using the VNA.

At first, a $25 \mathrm{~mm}$ diameter dielectric pipe and a thin conductive $2 \mathrm{~mm}$ width strip have been probed. As shown in the soil cross-section of Figure 13a, the pipe has been filled with water and the strip has been vertically positioned, thus its lateral dimension is $2 \mathrm{~mm}$, and its height is $10 \mathrm{~mm}$. The B-scans in both parallel and mirror polarizations are presented in Figures $13 \mathrm{~b}$ and $13 \mathrm{c}$ respectively. The time zero has not been corrected as the soil permittivity is not a priori precisely known, thus the arrival time of the clutter will serve as a reference to the arrival times of the hyperbola apexes. Comparing to the experimental WARR measurements in Figures $6 \mathrm{~b}$ and $6 \mathrm{~d}$, we remark that a few ground waves superimposed the reflected waves mostly in the parallel configuration. The arrival times of the clutter has been estimated to 4 and $4.2 \mathrm{~ns}$ in the parallel and mirror configurations respectively. Consequently, the time difference observed in the mirror configuration between the arrival of the clutter and the hyperbola apex is close to $1 \mathrm{~ns}$, that corresponds to a real permittivity value of 3 . This value has been confirmed when fitting both hyperbolas associated with the two buried objects. The probing of this soil structure highlights that the TM polarizations is preferred to detect a pipe filled with water and a very thin strip. The width of the objects cannot be estimated because of the significant lateral dimension of the radar as compared to their sizes, however we can observe that the strip gives a narrower hyperbola signature. 


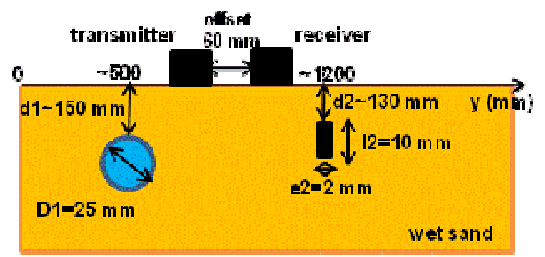

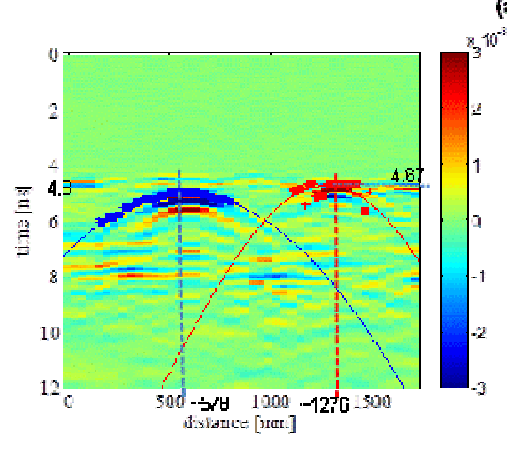

(b)

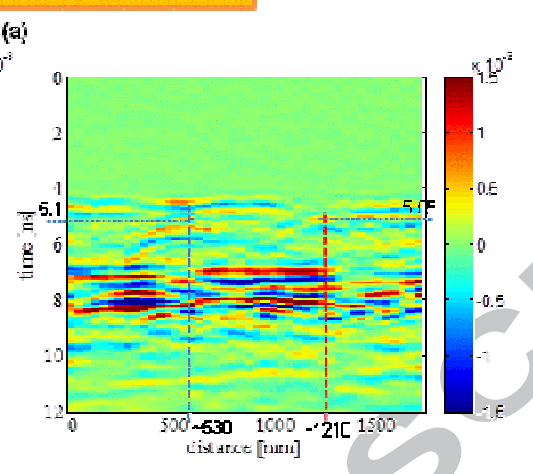

(c)

Figure 13

This soil structure has been reconsidered to include an air-filled pipe with air and the strip positioned horizontally a visualized in Figure 14a. From the B-scans presented in Figures 14b and $14 \mathrm{c}$, we notice that the arrival times associated with the apexes in both polarizations appear similar to the previous results. Furthermore, we remark that the TM polarization is not able to detect the air-filled pipe; this result agrees with the analysis of the SWs made from Figure $8 \mathrm{~d}$. The detection of the strip having a $1 \mathrm{~mm}$ lateral dimension is now possible using both polarizations.

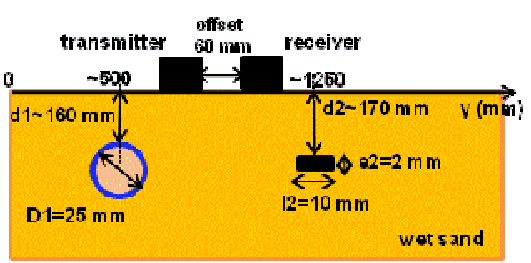

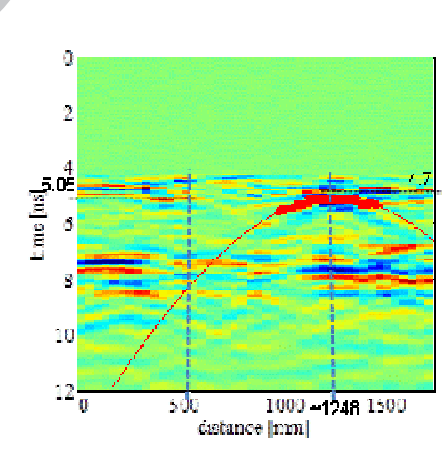

(b)

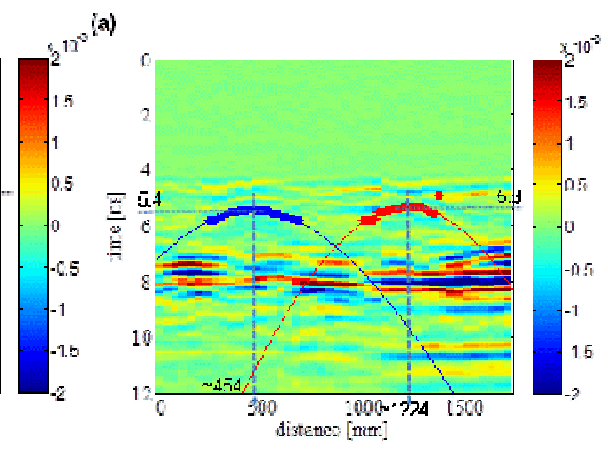

(c)

Figure 14 
A $20 \mathrm{~mm}$ diameter nylon pipe and a $12 \mathrm{~mm}$ conductive pipe have been now buried in the sand box as illustrated in Figure 15a. From B-scans in Figures 15b and 15c, we notice that when the dielectric contrast between the soil and the buried object is weak, the TM polarization is not able to make a detection. However, the TE polarization reveals a non contrasted hyperbola. According to previous results, the conductive pipe is better detected in the TM polarization. Because of the lack of contrast in this experiment, we have only plotted hyperbolas using the analytical modeling. The arrival times agree with those obtained in previous experimental radargrams.
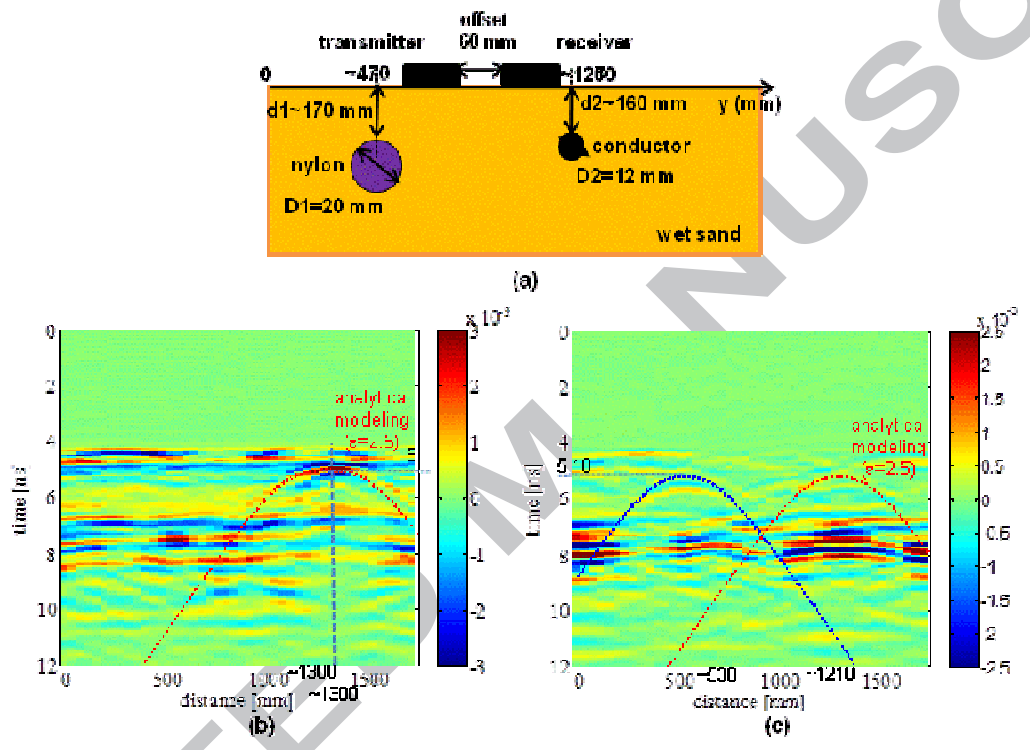

Figure 15

Finally, two canonical cracks filled with carboard have been made into the sand as shown in Figure 16a. Their average width from the left and the right has been estimated to $18 \mathrm{~mm}$ and their height is 75 and $150 \mathrm{~mm}$ respectively. Raw B-scans associated with both TM and TE polarizations presented in Figures $16 \mathrm{~b}$ and $16 \mathrm{c}$ respectively show that the hyperbolas have a slope corresponding to the propagation in the sand; the time zero has been corrected using the WARR B-scan in Figure $6 \mathrm{~b}$ and $6 \mathrm{~d}$. We remark that the hyperbolas appear with a weak contrast in the B-scans, and they are more detectable in the mirror configuration. This observation has been confirmed by the theoretical raw B-scan associated with two air-filled cracks at position $\mathrm{y}=-442.5$ and $162.5 \mathrm{~mm}$ visualized in Figure 17 in the case of the mirror configuration (common soil $\varepsilon_{s}^{\prime}=5.5, \sigma=0.01{\mathrm{~S} . \mathrm{m}^{-1}}^{2}$ ) in this case the arrival time difference has been estimated to $0.5 \mathrm{~ns}$ that corresponds to the analytical modeling. 

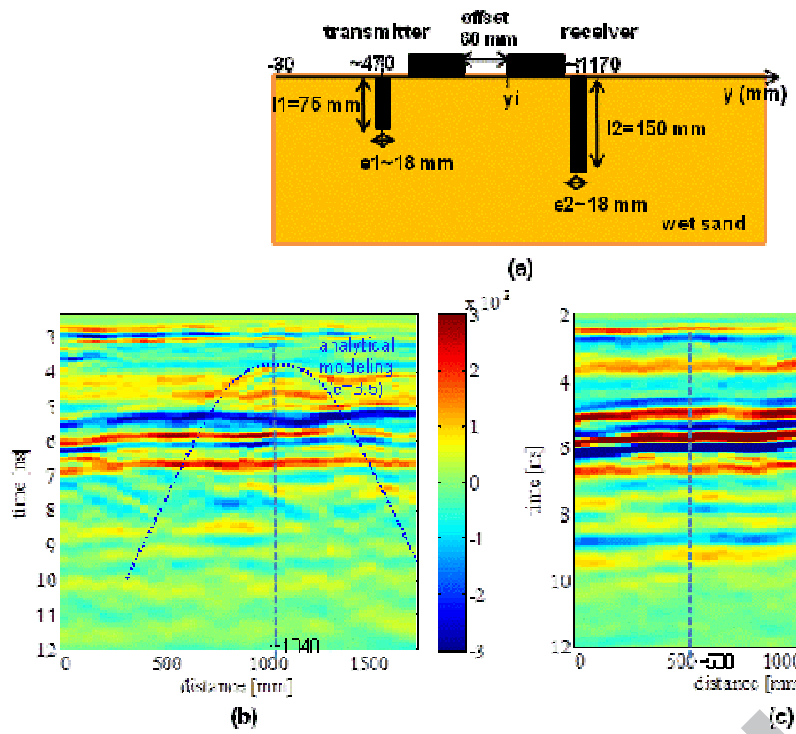

(a)

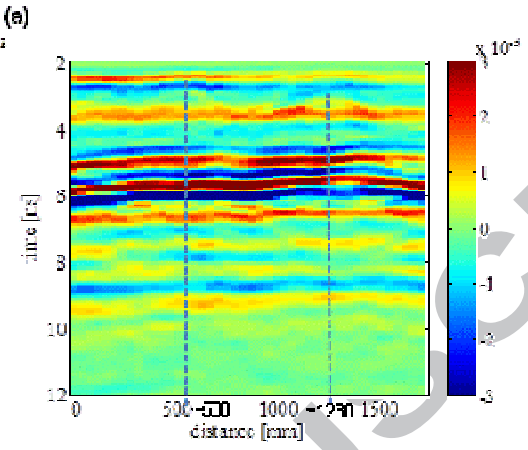

Figure 16

(c)

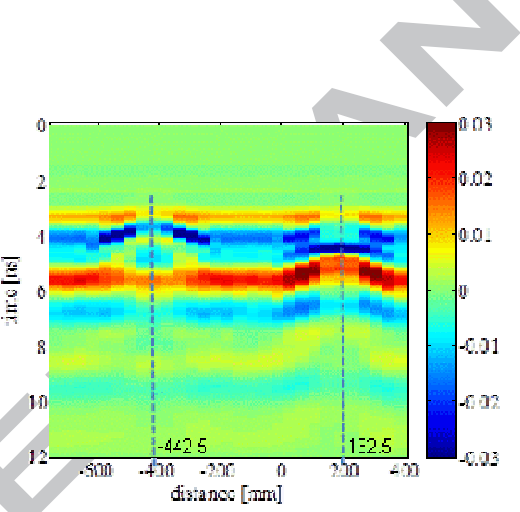

Figure 17

\section{Conclusion}

A parametric study associated with a novel compact UWB ground-coupled GPR radar operating in the frequency band $[0.46 ; 4] \mathrm{GHz}$ is presented in this work. This study has allowed to first characterize the radiating characteristics of each antenna, and then the radar system itself considering the two polarizations TM and TE. The performance of the GPR system in detecting very long buried dielectric or conductive objects has been particularly considered by comparing FDTD full-wave simulations and experimental results. The results highlight that the polarization diversity allows to discriminate the dielectric contrast between the buried object and the surrounding medium. Thus, the TM polarization is preferred to detect water-filled (higher real permittivity than the soil) or conductive pipes, and the TE polarization appears suitable for detecting air-filled (lower real permittivity than the soil) 
pipes. The polarizations of the transmitting and receiving antennas (co-polarization or cross polarization) that play a significant role in the detection of long object can play a significant role in improving the signal to clutter ratio [15-17]. First studies [16-17] need to be deepened to characterize the clutter for different target orientations with respect to the incident electric field and dielectric characteristics. This characterization will facilitate the reduction of the clutter in a given polarization. In subsequent studies soon proposed for publication, the authors have developed a semi-automatic algorithm for hyperbola detection based on template matching in the presence of overlapping signatures to detect a specific hyperbola pattern that has been used as a reference in the similarity measure. Afterwards, the parametric analysis of each hyperbola associated with the first arrival times has allowed to obtain estimates of the depth, size and soil real permittivity. Further studies will be focused on the sounding of the subsurface of our novel test site that will be built in the project Sense-City [18]. This subsurface will contain several buried utilities with different shapes (pipes and strips filled) and dielectric characteristics (conductor, air, and water) buried in parallel trenches. In particular, the influence of the polarization and the dielectric contrast of the objects with the surrounding medium will be studied.

\section{Acknowledgments}

The authors are grateful to the Sense-city project leaders who have accepted to bury utilities for research studies with GPR systems.

\section{References}

[1] D.J. Daniels, Ground penetrating radar. $2^{\text {nd }}$ edition: The IEE, London, 2004.

[2] J.L. Porsani, E. Slob, R.S. Lima, D.N Leite, Comparing detection and location performance of perpendicular and parallel broadside GPR antenna orientation. J. Applied Geophysics 70 (2010) 1-8.

[3] G.P. Tsoflias, J.P Van Gestel, P. Stoffa, D.D. Blankenship, M. Sen, Vertical fracture detection by exploiting the polarization properties of ground-penetrating radar signals. Geophysics 69 (3) (2004) 803-810.

[4] J. Becker, D. Filipovic, H. Schantz, S.Y. Suh, Ultra-wideband antennas, International Journal of Antennas and Propagation, Special Issue, Hindawi Publishing 2008. 
[5] B. Allen, M. Dohler, E. Okon, W. Malik, A. Brown, D. Edwards, Ultra Wideband Antennas and Propagation for Communications, Radar and Imaging, Wiley Ed 2007.

[6] C.J. Wu, I.F. Chen, C.M. Peng, A dual polarization bow-tie slot antenna for broad band communications. Progress in Electromagnetics Research Symposium Proceedings (PIERS), Marrakesh, Marocco, 20-23 March 2011 217-221.

[7] G. Zheng, A.A. Kishk, A.W. Glisson, A.B. Yakovlev, Slot antenna fed by a CPW line with tapered transition, Microwave and Opt. Technol. Letters 38 (6) (2003) 465-467.

[8] S.W. Qu, C.L. Ruan, Effects of round corners on bowtie antennas, PIER 57 (2006) 179195.

[9] S.H. Kim, L. Wen, H.W. Ko, D.H Park, B.C. Ahn, A technique for broadbanding the CPW-fed bowtie slot antenna, Journal of the Korea Electromagnetic Engineering Society 5 (1) (2005) 14-20.

[10] G.E. Atteia, A.A. Shaalan, Wideband partially-covered bowtie antenna for groundpenetrating radars, PIER 71 (2007) 211-226.

[11] D.C. Nobes, A.P. Annan, Broadside versus end-fire radar response: some simple illustrative examples, Proc. SPIE, Eighth International Conference on Ground Penetrating Radar (2000) 696-701.

[12] S. Shihab, W. Al-Nuaimy, Radius estimation for cylindrical objects detected by ground penetrating radar, Sensing and Imaging: An International Journal 6 (2) (2005) 151-166.

[13] S.J. Radzevicius, J.J. Daniels, Ground penetrating radar polarization and scattering from cylinders, J. Applied Geophys., 45 (2000) 111-125.

[14] C.A. Balanis, Advanced engineering electromagnetic, John Wiley and Sons 1989

[15] T. Khuut, Application of Polarimetric GPR to detection of subsurface objects, PhD Thesis, Tohoku University, 2009.

[16] C.C. Chen, Fully-polarimetric ground penetrating radar application, IEEE Antennas and Propagation Society International Symposium, 2001.

[17] E. Tebchrany, F. Sagnard, V. Baltazart, J.P. Tarel, X. Dérobert, Assessement of statistical-based clutter reduction techniques on ground-coupled GPR data for the detection of buried objects and cracks in soil, $15^{\text {th }}$ International Conference on Ground Penetrating Radar (GPR), 30 June-4 July 2014, Brussels (Belgium).

[18] http://www.sense-city.univ-paris-est.fr/index.php 


\section{Figure captions}

Figure 1: Geometry of the bowtie slot antenna (a) top view, (b) tappered CPW feed line, and (c) antenna shielded by a back-cavity filled with a layered radar absorbing material

Figure 2: Radiation properties of the bowtie slot antenna: (a) Comparison between theoretical and experimental S11 versus frequency; (b) E-field distribution in the TM and TE planes, and (c) radiation patterns of the shielded antenna at $800 \mathrm{MHz}$

Figure 3: The parallel (TM polarization) and mirror (TE polarization) configurations modeled under FDTD simulations

Figure 4: The experimental outdoor setup filled with wet sand in both (a) parallel, and (b) mirror configurations

Figure 5: FDTD simulation results associated with WARR probing on a common soil ( $\left.\varepsilon_{s}^{\prime}=5.5, \sigma=0.01 S . m^{-1}\right)$ in the frequency and time domains in both parallel (a, b) and mirror (c, d) configurations

Figure 6: Experimental results associated with WARR probing on the sandy box (square Perichaux, Paris $\left.15^{\text {th }}\right)$ in the frequency and time domains in both parallel $(\mathrm{a}, \mathrm{b})$ and mirror (c, d) configurations

Figure 7: The simplified analytical modeling based on the ray-tracing theory: (a) cross-view of the soil including a cylindrical pipe, and (b) plots of hyperbolas considering the dimensions of the experimental GPR systems in both configurations of polarization $(\mathrm{d}=150 \mathrm{~mm}, \mathrm{R}=10$ $\mathrm{mm}$, offset=60 $\mathrm{mm}$ )

Figure 8: Influence of the pipe radius and the real permittivity on the scattering width (SW) as a function of the frequency in the case of a conductive $(a, b)$ and a dielectric air-filled cylinder (c,d)

Figure 9: Probing issued from FDTD simulations (soil $\varepsilon^{\prime}=5.5 ; \sigma=0.01 \mathrm{~S} / \mathrm{m}$ ) of an opened rectangular crack filled with air in the (a) parallel, and (b) mirror configurations; B-scans associated with (c) the parallel and (d) the mirror configurations

Figure 10: (a) Comparison of waveforms at the apex (median value removed, soil $\varepsilon^{\prime}=5.5$; $\sigma=0.01 \mathrm{~S} / \mathrm{m}$ ) issued from the probing of the rectangular air-filled crack in the parallel and mirror configurations; (b) Comparison of hyperbolas extracted from numerical B-scans (soil $\varepsilon^{\prime}=5.5 ; ; \sigma=0.01 \mathrm{~S} / \mathrm{m}$ ) and associated with a rectangular and a $\mathrm{V}$-crack with $5 \mathrm{~mm}$ width

Figure 11: Probing issued from FDTD simulations of a buried ( soil $\varepsilon^{\prime}=3.5 ; \sigma=0.01 \mathrm{~S} / \mathrm{m}$ ) conductive cylindrical pipe in (a) the parallel, and (b) mirror configurations; B-scans associated with (c) the parallel and (d) the mirror configurations

Figure 12: Comparison of the theoretical waveforms straight above the cylindrical pipe at the hyperbola apex associated with the parallel and mirror configurations 
Figure 13: (a) Experimental setup associated with the probing of a water-filled cylindrical pipe and a vertical conductive strip; B-scans (with median value removed) associated with (b) the parallel and (c) mirror configurations

Figure 14: (a) Experimental setup associated with the probing of an air-filled cylindrical pipe and a horizontal conductive strip; B-scans (with median value removed) associated with (b) the parallel and (c) mirror configurations

Figure 15: (a) Experimental setup associated with the probing of a solid nylon pipe and a conductive pipe; B-scans associated with (b) the parallel (raw image) and (c) mirror (with medium value removed) configurations

Figure 16: (a) Experimental setup associated with the probing of two opened $18 \mathrm{~mm}$ width cracks with heights 75 and $150 \mathrm{~mm}$; B-scans (raw images) associated with (b) the parallel and (c) mirror configurations

Figure 17: Theoretical B-scan (raw image) obtained from FDTD simulations of two opened $18 \mathrm{~mm}$ width cracks with heights 75 and $150 \mathrm{~mm}$ in a common soil $\varepsilon_{s}^{\prime}=5.5, \sigma=0.01{\mathrm{~S} . \mathrm{m}^{-1}}^{-}$ 

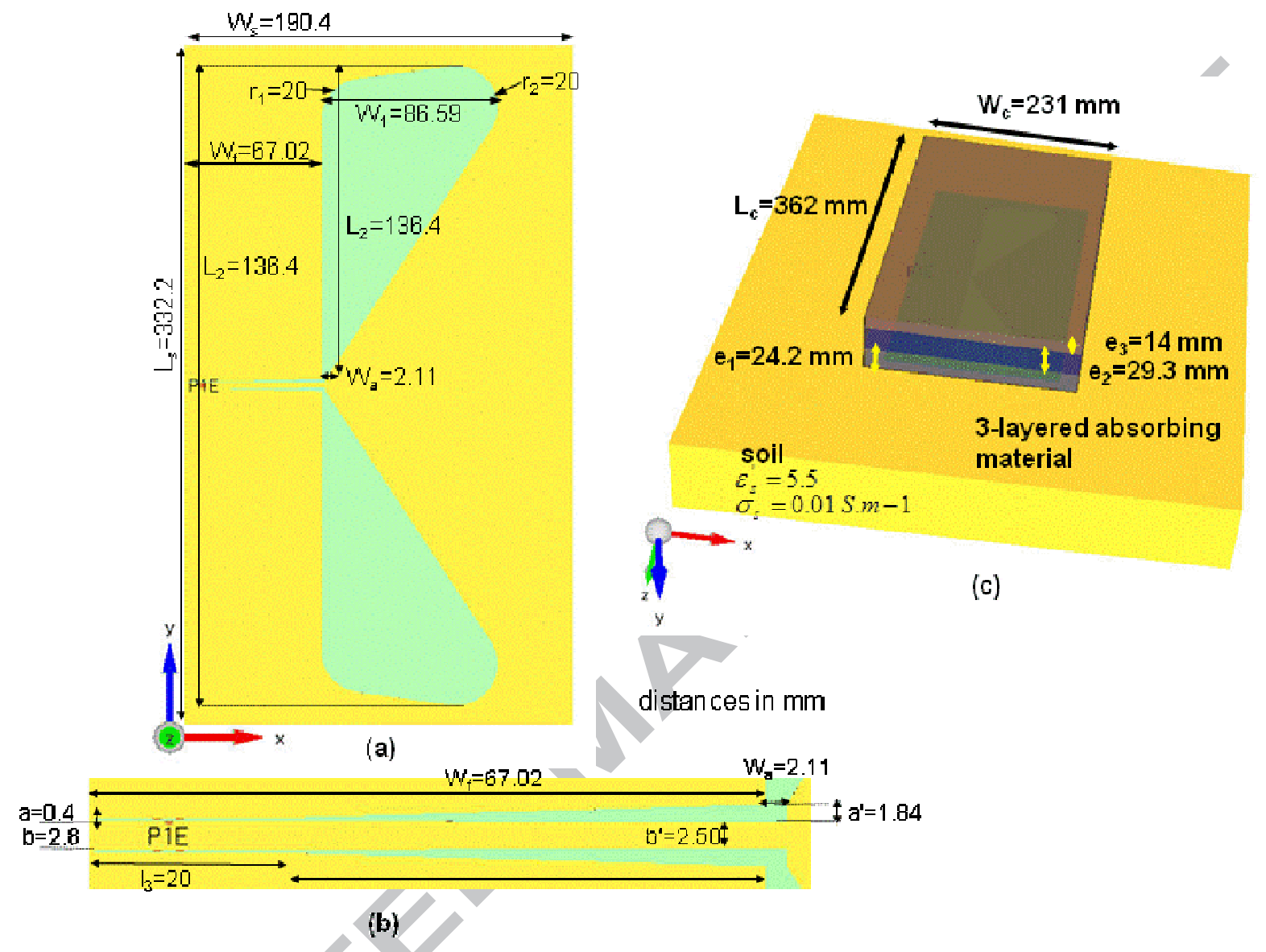

Fig. 1 


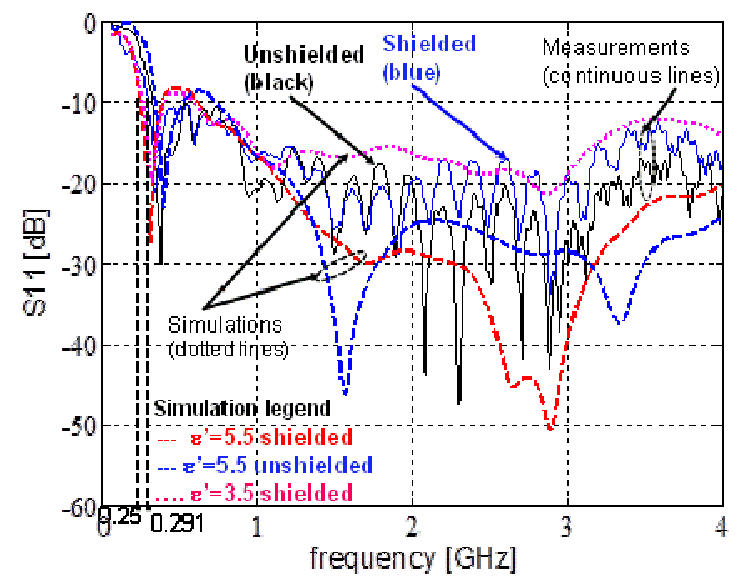

(a)

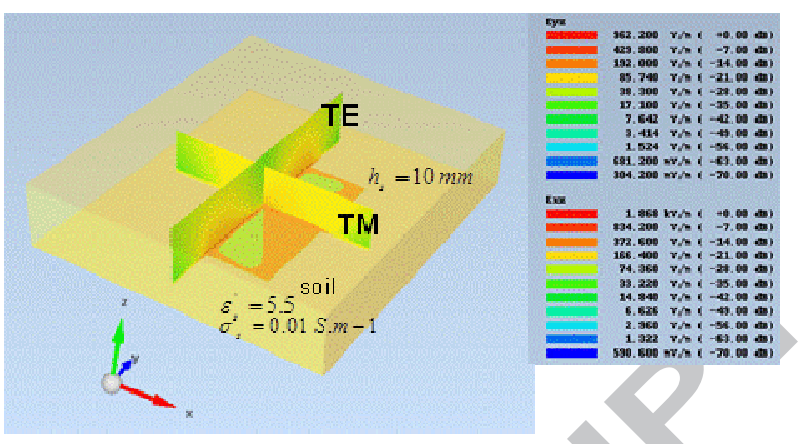

(b)

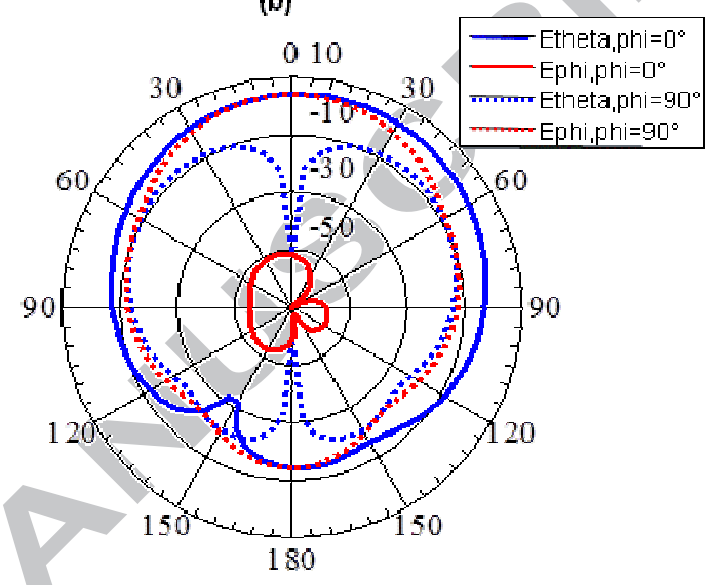

(c)

Figure 2 


\section{Highlights}

- Design of a shielded UWB bowtie slot antenna at frequencies $460 \mathrm{MHz}$ to beyond $4 \mathrm{GHz}$

- 3D FDTD simulations of a GPR system as a function of various parameters

- Imaging of dielectric and conductive pipes using polarization diversity

- Comparison of theoretical and experimental B-scans with buried pipes in a sand 